\title{
The Imaging X-ray Polarimetry Explorer (IXPE): technical overview
}

Stephen L. O'Dell ${ }^{\text {a }}$, Luca Baldini ${ }^{\mathrm{b}}$, Ronaldo Bellazzini ${ }^{\mathrm{b}}$, Enrico Costa ${ }^{\mathrm{c}}$,

Ronald F. Elsner ${ }^{a}$, Victoria M. Kaspi ${ }^{\mathrm{d}}$, Jeffery J. Kolodziejczak ${ }^{\mathrm{a}}$, Luca Latronico ${ }^{\mathrm{e}}$,

Herman L. Marshall ${ }^{\mathrm{f}}$, Giorgio Matt ${ }^{\mathrm{g}}$, Fabio Muleri ${ }^{\mathrm{c}}$, Brian D. Ramsey ${ }^{\mathrm{a}}$, Roger W. Romani ${ }^{\mathrm{h}}$, Paolo Soffitta ${ }^{c}$, Allyn F. Tennant ${ }^{a}$, Martin C. Weisskopf ${ }^{a}$,

D. Zachery Allen ${ }^{\mathrm{i}}, \mathrm{Fabrizio} \mathrm{Amici}^{\mathrm{c}}$, L. Angelo Antonelli ${ }^{\mathrm{j}}$, Spencer Antoniak ${ }^{\mathrm{i}}$, Primo Attina ${ }^{\mathrm{k}}$, Matteo Bachetti ${ }^{1}$, Mattia Barbanera ${ }^{b}$, Wayne H. Baumgartner ${ }^{a}$, Jeff Bladt ${ }^{i}$, Stephen D. Bongiorno ${ }^{a}$,

Fabio Borotto ${ }^{\mathrm{e}}$, Alessandro Brez ${ }^{\mathrm{b}}$, Thomas Brooks ${ }^{\mathrm{a}}$, Scott Bussinger ${ }^{\mathrm{i}}$, H. Kyle Bygott ${ }^{\mathrm{i}}$,

Elisabetta Cavazzuti ${ }^{\mathrm{m}}$, Marco Ceccanti ${ }^{\mathrm{b}}$, Saverio Citraro ${ }^{\mathrm{b}}$, William D. Deininger ${ }^{\mathrm{i}}$, Ettore Del Monte ${ }^{c}$, Kurtis L. Dietz ${ }^{a}$, Niccolò Di Lalla ${ }^{c}$, Giuseppe Di Persio ${ }^{c}$, Immacolata Donnarumma ${ }^{\mathrm{m}}$, Jennifer Erickson ${ }^{\mathrm{i}}$,Yuri Evangelista ${ }^{\mathrm{c}}$, Sergio Fabiani ${ }^{\mathrm{c}}$, Riccardo Ferrazzoli ${ }^{c}$, Michelle Foster ${ }^{a}$, Manuela Giusti ${ }^{c}$, Shuichi Gunji ${ }^{\mathrm{n}}$, Larry Guy ${ }^{\mathrm{i}}$, Sandra Johnson ${ }^{i}$, William Kalinowski ${ }^{i}$, Anthony R. Kelley ${ }^{a}$, Kiranmayee Kilaru ${ }^{\circ}$, Carlo Lefevre ${ }^{c}$, Simone Maldera ${ }^{\mathrm{e}}$, Alberto Manfreda ${ }^{\mathrm{b}}$, Marco Marengo ${ }^{\mathrm{e}}$, James Masciarelli ${ }^{\mathrm{i}}$, Mike McEachen ${ }^{\mathrm{p}}$, Paolo Mereu ${ }^{\mathrm{e}}$, Massimo Minuti ${ }^{\mathrm{b}}$, Mark A. Mitchell ${ }^{\mathrm{a}}$, Scott Mitchell ${ }^{\mathrm{i}}$, Ikuyuki Mitsuishi ${ }^{\mathrm{q}}$, Alfredo Morbidini ${ }^{\mathrm{c}}$, Federico Mosti ${ }^{\mathrm{e}}$, Hikmat Nasimi ${ }^{\mathrm{b}}$, Barbara Negri ${ }^{\mathrm{m}}$,

Leonardo Orsini ${ }^{b}$, Darren Osborne ${ }^{r}$, Steven D. Pavelitz ${ }^{a}$, Christina Pentz ${ }^{i}$, Matteo Perri ${ }^{j}$, Melissa Pesce-Rollins ${ }^{b}$, Colin Peterson ${ }^{i}$, Raffaele Piazzolla ${ }^{c}$, Stefano Pieraccini ${ }^{\text {s }}$, Maura Pilia ${ }^{1}$, Michele Pinchera ${ }^{b}$, Simonetta Puccetti ${ }^{m}$, Jaganathan Ranganathan ${ }^{\circ}$, Tim Read $^{i}$, Alda Rubini ${ }^{c}$,

Francesco Santoli ${ }^{c}$, Paolo Sarra ${ }^{\text {s }}$, Sarah Schindhelm ${ }^{\mathrm{i}}$, Andrea Sciortino ${ }^{\mathrm{s}}$, Chris Seckar ${ }^{\mathrm{i}}$,

Carmelo Sgrò ${ }^{b}$, Brian T. Smith ${ }^{\mathrm{i}}$, Gloria Spandre ${ }^{\mathrm{b}}$, Chet O. Speegle ${ }^{\mathrm{a}}$, Toru Tamagawa ${ }^{\mathrm{t}}$, Marcello Tardiola ${ }^{\mathrm{e}}$, Antonino Tobia ${ }^{\mathrm{c}}$, Alessia Tortosa ${ }^{\mathrm{c}}$, Alessio Trois ${ }^{1}$, Bruce Weddendorf ${ }^{u}$, Jeff Wedmore ${ }^{i}$, Davide Zanetti ${ }^{\mathrm{b}}$

${ }^{a}$ NASA Marshall Space Flight Ctr., Huntsville, AL 35812, USA

${ }^{b}$ Istituto Nazionale di Fisica Nucleare - Pisa, Largo Bruno Pontecorvo 3, 56127 Pisa (PI), Italy

${ }^{\mathrm{c}}$ INAF Istituto di Astrofisica e Planetologia Spaziali, Via del Fosso del Cavaliere 100, 00133 Roma (RM), Italy

${ }^{\mathrm{d}}$ McGill University, 3600 University Street, Montreal QC, H3A 2T8, Canada

${ }^{\mathrm{e}}$ Istituto Nazionale di Fisica Nucleare - Torino, Via Pietro Giuria 1, 10125 Torino (TO), Italy

${ }_{\mathrm{f}}^{\mathrm{f}}$ Massachusetts Institute of Technology, 77 Massachusetts Avenue, Cambridge, MA 02139, USA

${ }^{\mathrm{g}}$ Università degli Studi Roma Tre, Via della Vasca Navale 84, 00146 Roma (RM), Italy

${ }^{\mathrm{h}}$ Stanford University, 382 Via Pueblo Mall, Stanford, CA 94305-4060, USA

${ }^{i}$ Ball Aerospace, 1600 Commerce Street, Boulder, CO 80301 USA

${ }^{j}$ INAF Osservatorio Astronomico di Roma, Via Frascati 33, 00078 Monte Porzio Catone (RM), Italy

${ }^{\mathrm{k}}$ INAF Osservatorio Astrofisico di Torino, Strada Osservatorio 20, 10025 Pino Torinese (TO), Italy

${ }^{1}$ INAF Osservatorio Astronomico di Cagliari, Via della Scienza 5, 09047 Selargius (CA), Italy

${ }^{m}$ Agenzia Spaziale Italiana, Via del Politecnico, 00133 Roma (RM), Italy

${ }^{\mathrm{n}}$ Yamagata University, 1-4-12 Kojirakawa-machi, Yamagata-shi, 990-8560, Japan

${ }^{\circ}$ USRA Marshall Space Flight Ctr., Huntsville, AL 35812, USA

p Northrop Grumman Innovation Systems, 600 Pine Avenue, Goleta, CA 93117, USA

* Contact author (SLO): stephen.1.odell@nasa.gov; voice +1 (256) 961-7776; fax +1 (256) 961-7522

Postal address: NASA/MSFC/ST12; 320 Sparkman Drive NW; Huntsville, AL 35805-1912, USA 


\author{
q Nagoya University, Furo-cho, Chikusa-ku, Nagoya, 464-8602, Japan \\ ${ }^{\mathrm{r}}$ Lab. For Atmospheric \& Space Physics, 1234 Innovation Drive, Boulder, CO 80303, USA \\ ${ }^{s}$ OHB Italia SpA, Via Gallarate 150, 20151 Milano (MI), Italy \\ ${ }^{t}$ RIKEN Nishina Ctr., 2-1 Hirosawa, Wako, Saitama 351-0198, Japan \\ u Weddendorf Design, 14060 Valley Vista Drive, Huntsville, AL 35803, USA
}

\begin{abstract}
The Imaging X-ray Polarimetry Explorer (IXPE) will expand the information space for study of cosmic sources, by adding linear polarization to the properties (time, energy, and position) observed in x-ray astronomy. Selected in 2017 January as a NASA Astrophysics Small Explorer (SMEX) mission, IXPE will be launched into an equatorial orbit in 2021. The IXPE mission will provide scientifically meaningful measurements of the x-ray polarization of a few dozen sources in the 2-8 $\mathrm{keV}$ band, including polarization maps of several x-ray-bright extended sources and phase-resolved polarimetry of many bright pulsating $\mathrm{X}$-ray sources.
\end{abstract}

Keywords: X-ray astronomy, polarimetry, missions, grazing-incidence optics, gas pixel detectors

\title{
1. INTRODUCTION
}

The Imaging X-ray Polarimetry Explorer (IXPE) ${ }^{1,2}$ will be the first astronomy mission dedicated to $\mathrm{x}$-ray polarimetry and the first to perform x-ray polarimetric imaging. As such, it will expand the information space for study of celestial x-ray sources, adding linear polarization degree and position angle (or equivalently, Q and U Stokes parameters) to temporal, spectral, and imaging data. This polarization information provides constrains on astrophysical models involving magnetic fields or scattering, which temporal and spectral data cannot of themselves provide.

Over four decades ago, the crystal polarimeters aboard the Orbiting Solar Observatory 8 (OSO-8) obtained the first and thus far only measurements at high statistical significance, of the x-ray polarization of a cosmic source- the Crab Nebula. ${ }^{3,4}$ In terms of integration time required to reach a given polarization sensitivity, IXPE is nearly two-orders of magnitude faster than the OSO-8 polarimeter. Such sensitivity in a dedicated polarimetry mission means that IXPE will make scientifically meaningful measurements of the x-ray polarization properties of a few dozen sources, drawn from several categories of astrophysical sources, during its first year of operation.

The current paper provides a technical overview of the IXPE mission (§2), its flight system (§3), and its ground system (§4). A more detailed technical description is in preparation.

\section{MISSION}

Following a successful step-1 proposal submitted in 2014 and a step-2 proposal (Concept Study Report, CSR) submitted in 2016, NASA's Science Mission Directorate (SMD) selected IXPE in 2017 January, as an Astrophysics Small Explorer (SMEX) mission. NASA Marshall Space Flight Center (MSFC) manages the IXPE Project, reporting to the Explorers Program Office at Goddard Space Flight Center (GSFC). IXPE is a NASA mission in partnership with the Italian space agency, Agenzia Spaziale Italiana (ASI).

Since selection, the IXPE Team has matured the mission architecture and design, to enable accomplishing the science objectives of the mission. Section 2.1 outlines the science objectives; Section 2.2 summarizes the mission architecture and technical capabilities; and Section 2.3 relates the responsibilities of the mission partners.

\subsection{Science objectives}

IXPE's essential science goal is to expand the understanding of high-energy astrophysical processes and sources, in support of NASA's first scientific objective in astrophysics: "Discover how the universe works". Using x-ray polarimetry, IXPE will pursue the following specific goals: 
1. Constrain the radiation processes and detailed properties of different types of cosmic x-ray sources

2. Investigate general relativistic and quantum effects in extreme environments

3. Constrain the geometry and the emission mechanism(s) of active galactic nuclei (AGN) and of microquasars

4. Establish the geometry and strength of the magnetic field in magnetars

5. Constrain the geometry and origin of the x-radiation from radio pulsars

6. Learn how particles are accelerated in Pulsar Wind Nebulae and in (shell-type) Supernova Remnants (SNR)

IXPE scientists are formulating a two-year observing program with specific scientific objectives to address these goals. Besides information related to polarization direction, the IXPE instrument records arrival time, location, and pulseinvariant energy of each detected photon. Hence, for the brighter sources, IXPE can measure not only the integrated x-ray polarization at high statistical significance, but also the spectral dependence, phase dependence (for pulsed sources), or map (for extended sources) of the x-ray polarization.

\subsection{Mission description}

Figure 1 synopsizes the architecture and technical capabilities of the IXPE mission, which the remainder of this paper will sketch in somewhat more detail. A comprehensive report on the IXPE mission will appear elsewhere.

Following a 1-month commissioning phase, the baseline IXPE mission will conduct science operations for 2 years. During this period, all scientific data from the IXPE observing program will be publicly released through NASA's High-Energy Astrophysics Science Archive Research Center (HEASARC) at GSFC. Pending NASA approval, IXPE will continue beyond the first 25 months, initiating a guest observer (GO) program to be administered by the HEASARC.

IXPE's orbit will be nominally equatorial and circular, at a 540-km altitude (minimum). ASI will contribute use of its Malindi (Kenya) ground station ( $3^{\circ} \mathrm{S}$ latitude) as the primary contact for communications (command and telemetry) with the IXPE flight system (§3)-termed the "Observatory".

\begin{tabular}{|l|l|}
\hline Mission name & Imaging X-ray Polarimetry Explorer (IXPE) \\
\hline Mission category & NASA Astrophysics Small Explorer (SMEX) \\
\hline Operational phase & 2021 launch, 2 years following 1 month commissioning, extension possible \\
\hline Orbital parameters & Circular at 540-620 km altitude, equatorial; one ground station near equator \\
\hline Spacecraft features & 3-axis stabilized pointing (non-propellant), GPS time and position \\
\hline Science payload & 3 x-ray telescopes, 4.0-m focal length (deployed), co-aligned to star tracker \\
\hline Telescope optics $(\times 3)$ & 24 monolithic (P+S surfaces) Wolter-1 electroformed shells, coaxially nested \\
\hline Telescope detector (×3) & Polarization-sensitive gas pixel detector (GPD) to image photo-electron track \\
\hline Polarization sensitivity & Minimum Detectible Polarization (99\% confidence) MDP 99 5.5\%, 0.5-mCrab, 10 days \\
\hline Spurious modulation & $<0.3 \%$ systematic error in modulation amplitude for unpolarized source \\
\hline Angular resolution & $<30$-arcsec half-power diameter (HPD) \\
\hline Field of view (FOV) & $\approx 10$-arcmin diameter overlapping FOV of 3 detectors' polarization-sensitive areas \\
\hline Energy band, resolution & 2-8 keV, $\approx 20 \% @ 5.9$ keV \\
\hline Timing accuracy & $\approx 20 \mu$ s, using GPS pulse-per-second signal and on-board clocks \\
\hline X-ray calibration & Telescopes (optics \& detector separately, combined) on-ground; detectors on-orbit \\
\hline
\end{tabular}

Figure 1. Mission description and technical capabilities.

The spacecraft bus ( $\$ 3.2)$ supplies the functionality to operate the satellite. This includes features to support operation of the flight system as an astronomical observatory: 3-axis stabilized pointing (non-propellent), star trackers, GPS receivers for time and position, etc. 
The scientific payload ( $\$ 3.1$ ) of the flight system constitutes essentially the entire x-ray telescope system. It comprises 3 $\mathrm{x}$-ray telescopes, each with $\mathrm{x}$-ray optics and a polarization-sensitive $\mathrm{x}$-ray detector that are separated by a shared deployable optical bench (boom), which establishes the 4-m focal length of the x-ray telescopes. Additional payload components support operation of the telescopes - including telescope alignment mechanisms, a metrology monitoring system, stray-Xray shielding, and thermal control of the detectors.

The telescopes are optimized for $\mathrm{x}$-ray polarimetry at 2-8 keV. In addition to measuring $\mathrm{x}$-ray polarization, the telescopes provide moderate angular resolution $(<0.5 \mathrm{arcmin})$ over a useful field of view $(\approx 10 \mathrm{arcmin})$, moderate spectral resolution $(\approx 20 \%$ at $5.9 \mathrm{keV})$, and excellent timing resolution $(\approx 20 \mu \mathrm{s})$. The polarization sensitivity of IXPE enables accomplishing the specific scientific objectives of the observing program within the 2-year baseline mission.

The IXPE Team will conduct an extensive x-ray calibration program on the ground to characterize the performance of each X-ray optic, each detector, and each telescope (paired optic and detector). In addition, each detector assembly includes a filter calibration wheel containing radioactive sources that produce $\mathrm{x}$-ray fluorescence lines for monitoring the detector performance on orbit. Both on-ground and on-orbit calibrations will utilize polarized sources and unpolarized sources.

\subsection{Mission partners}

As IXPE is a NASA mission in partnership with the Italian space agency ASI, several US and Italian institutions contribute to the technical and scientific preparation and execution of the mission. Figure 2 highlights the roles of the IXPE partners.

\begin{tabular}{|c|c|}
\hline $\begin{array}{l}\text { NASA Marshall Space Flight Center } \\
\text { PI team, project management, } \\
\text { SE and S\&MA oversight, mirror }\end{array}$ & 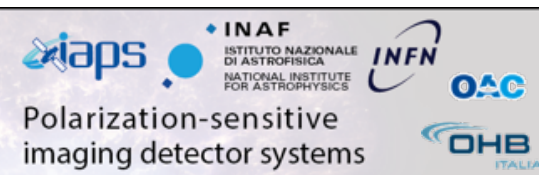 \\
\hline \multirow{2}{*}{$\begin{array}{l}\text { PI team, project management, } \\
\text { SE and S\&MA oversight, mirror } \\
\text { module fabrication, X-ray } \\
\text { calibration, science operations, } \\
\text { and data analysis and archiving }\end{array}$} & SP Mission operations \\
\hline & \multirow{2}{*}{ ROMA Stanford Scientific theory } \\
\hline Detect & \\
\hline & भix McGill \\
\hline \multirow{2}{*}{ Ball $\begin{array}{l}\text { Spacecraft, payload } \\
\text { structure, payload, } \\
\text { observatory I\&T }\end{array}$} & \\
\hline & |||- \\
\hline
\end{tabular}

Figure 2. Mission partners, including technical and scientific contributors.

NASA MSFC - home organization of the IXPE Principal Investigator (PI) - manages the IXPE Project and provides systems engineering (SE) and Safety and Mission Assurance (S\&MA) oversight. MSFC also designs and fabricates IXPE's x-ray optics - Mirror Module Assemblies (MMAs) - and performs x-ray testing and calibration of each MMA and of each telescope (MMA + detector) at its 100-m x-ray test facility. In addition, MSFC is responsible for the IXPE Science Operations Center (SOC), including software development for science data processing.

ASI oversees and funds the development of the IXPE instrument (detector system) and related research at multiple Italian institutions. In addition, ASI provides its Malindi (Kenya) facility as IXPE's primary ground station and it supports the SOC in software development for science data processing, through its Space Science Data Center (SSDC).

The Istituto Nazionale di Astrofisica (INAF) Istituto di Astrofisica e Planetologia Spaziali (IAPS) - home institute of the Italian Principal Investigator (IPI) - coordinates development of the instrument on behalf of ASI, providing overall management, systems engineering, product assurance, and x-ray calibration. Other INAF organizations - Osservatorio Astronomico di Cagliari (OAC) and Osservatorio Astrofisico di Torino-support INAF-IAPS in these activities.

The Istituto Nazionale di Fisica Nucleare (INFN) Pisa-home institute of the Italian Co-PI—is responsible for design, development, assembly, testing, and simulation of the detector units (DUs) for the IXPE instrument. In this activity, INFNTorino is supporting INFN-Pisa. 
OHB Italia SpA (OHB-I), under contract to ASI, is the industry partner for the IXPE instrument. It provides certain components of the DUs, engineering services, environmental testing, and some ground support equipment (GSE). In addition, OHB-I is responsible for the Detectors Service Unit (DSU), which controls all 3 DUs, receives and packages DU data, and communicates with the spacecraft's Integrated Avionics Unit (IAU).

Ball Aerospace - IXPE prime contractor - is responsible for the spacecraft bus, certain payload elements, integration and testing of the flight system, and engineering support. This support continues throughout orbital commissioning and operations; it includes oversight of the IXPE Mission Operations Center (MOC), at the University of Colorado's Laboratory for Atmospheric and Space Physics (LASP).

To supplement the scientific expertise within the x-ray optics and instrument groups, the IXPE Team includes Science CoInvestigators from Università Roma Tre, Stanford University, McGill University, and the Massachusetts Institute of Technology. Finally, a multi-national Science Advisory Team provides valuable astrophysics expertise for a diverse range of x-ray sources, which will help guide formulation of IXPE's observing program, as well as analysis and interpretation of science data obtained during the two-year baseline mission.

\section{FLIGHT SYSTEM}

Figure 3 displays the IXPE flight system — termed the "Observatory" - in three configurations. Upon being fully deployed for science observations, the Observatory provides three x-ray telescopes, with 4.0-m focal length, optimized for x-ray imaging $x$-ray polarimetry in the energy range $2-8 \mathrm{keV}$. In terms of functionality and for purposes of assembly and processing, the flight system comprises the payload (\$3.1) and the spacecraft (§3.2), as described below.
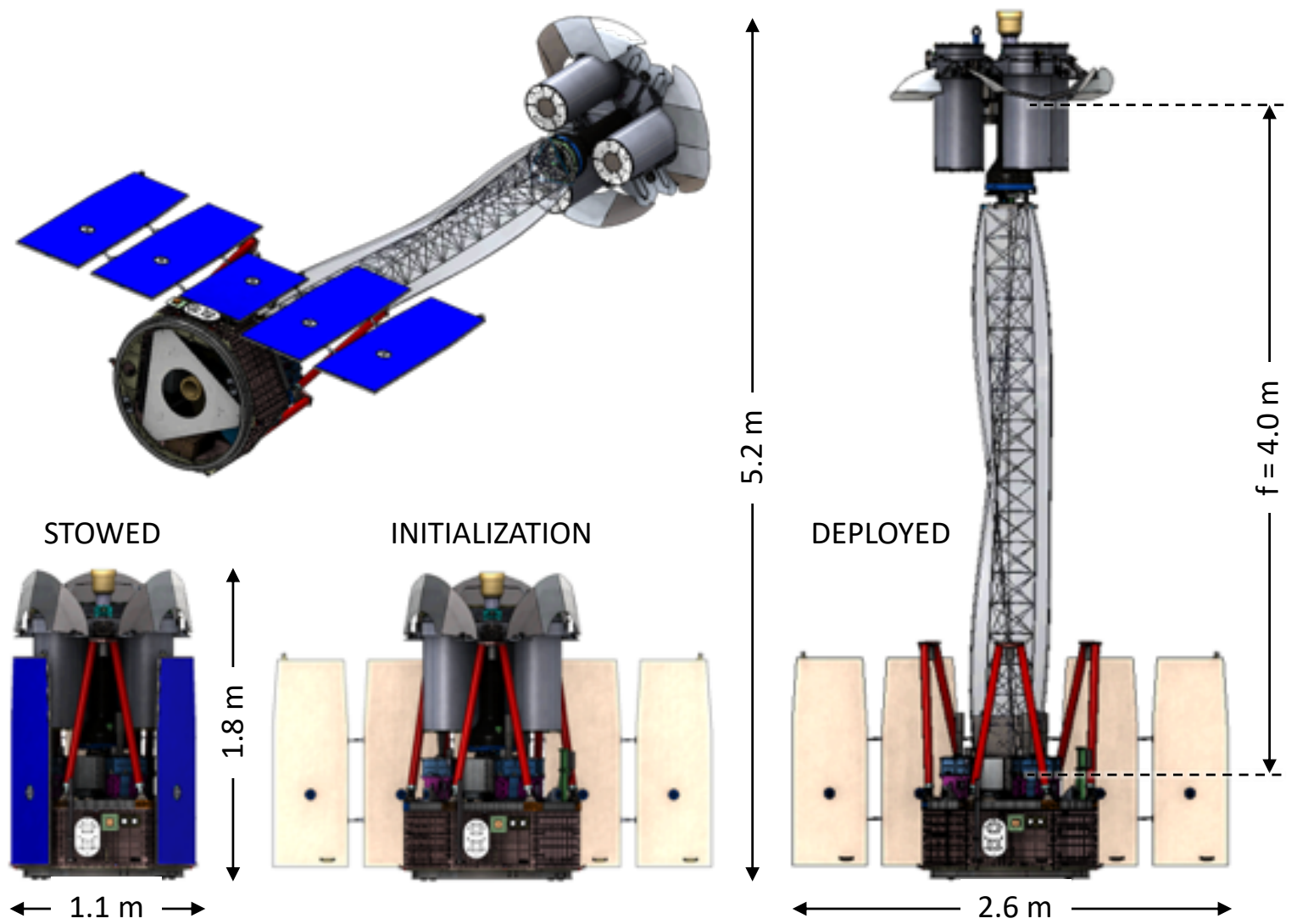

Figure 3. IXPE flight system - the Observatory-shown (left-to-right) in the stowed configuration for launch, in the initialization configuration for commencing spacecraft operations, and in the fully deployed configuration for science observations. The CAD image to the upper left provides another view of the Observatory in its fully deployed configuration. 


\subsection{Payload}

Figure 4 shows the IXPE payload, which constitutes the "Telescope System" of the Observatory. The Telescope System comprises the $\mathrm{x}$-ray optics (\$3.1.1), the $\mathrm{x}$-ray instrument (\$3.1.2), and other payload elements (\$3.1.3). A detailed $\mathrm{x}$-ray calibration program (\$3.1.4) will assess the performance of the x-ray optics and instrument (both separately and combined as telescopes), prior to delivery to Ball for alignment (\$3.1.5) and integration with other payload components and with the spacecraft (\$3.2).

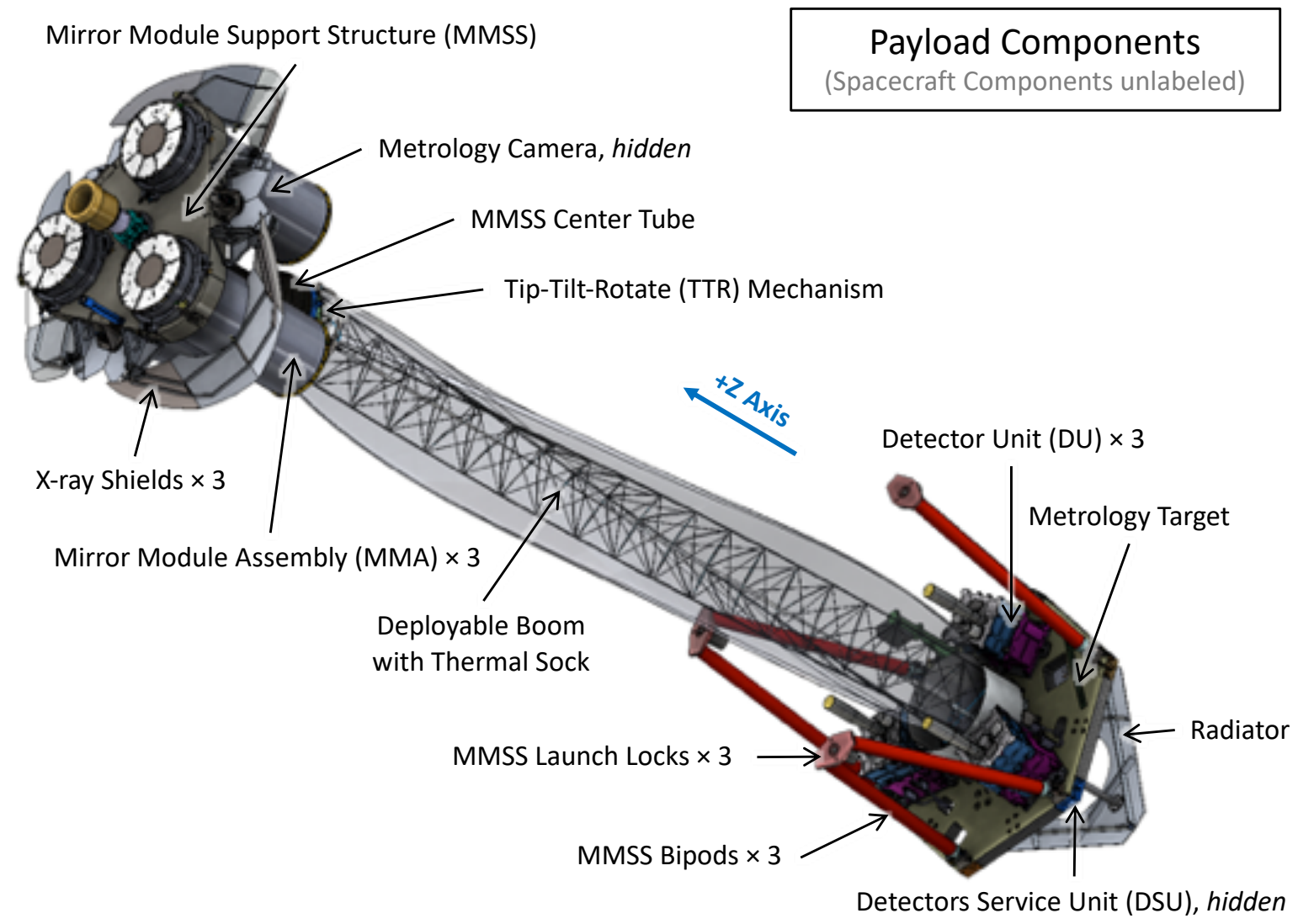

Figure 4. IXPE payload - the Telescope System - in its deployed state for science observations. Payload components are labeled; spacecraft components are not.

\subsubsection{X-ray optics}

Figure 5 illustrates the IXPE x-ray optics ${ }^{5}$ - three identical Mirror Module Assemblies (MMAs) — provided by NASA MSFC. The right two panels identify the MMA components. The thermal shields - contributed by Nagoya University (Japan) - and the outer housing tube, along with heaters and multilayer insulation (MLI) from Ball, stabilize the thermal environment within the MMA optical cavity. The optical cavity contains 24 (uncoated) electroformed nickel-cobalt (tworeflection) grazing-incidence shells. Each shell is approximately $600 \mathrm{~mm}(300 \mathrm{~mm}$ per surface) in length and designed to a $4001-\mathrm{mm}$ focal length. The 24 shells have diameters in the range $162-272 \mathrm{~mm}$, resulting in a $2.3-\mathrm{keV}$ effective area of about $200 \mathrm{~cm}^{2}$ per MMA, for the (uncoated) nickel-cobalt surfaces-ideal for response in IXPE's 2-8-keV band. The angular resolution of each MMA will be approximately 25" half-power diameter (HPD).

Each of the MMA's 24 shells is aligned to the (central) inner support tube and bonded into combs attached to the fore spider. In addition to supporting the central tube and the 24 shells, the fore spider provides a precision mechanical interface to the Mirror Module Support Structure (MMSS), shown in the left panel of Figure 5, along with other payload components (\$3.1.3 and Figure 4). At the opposite end of the MMA, the aft spider serves as a mechanical snubber, with combs that limit the amplitude of shell vibrations and thus preclude collisions of adjacent shells during launch. 

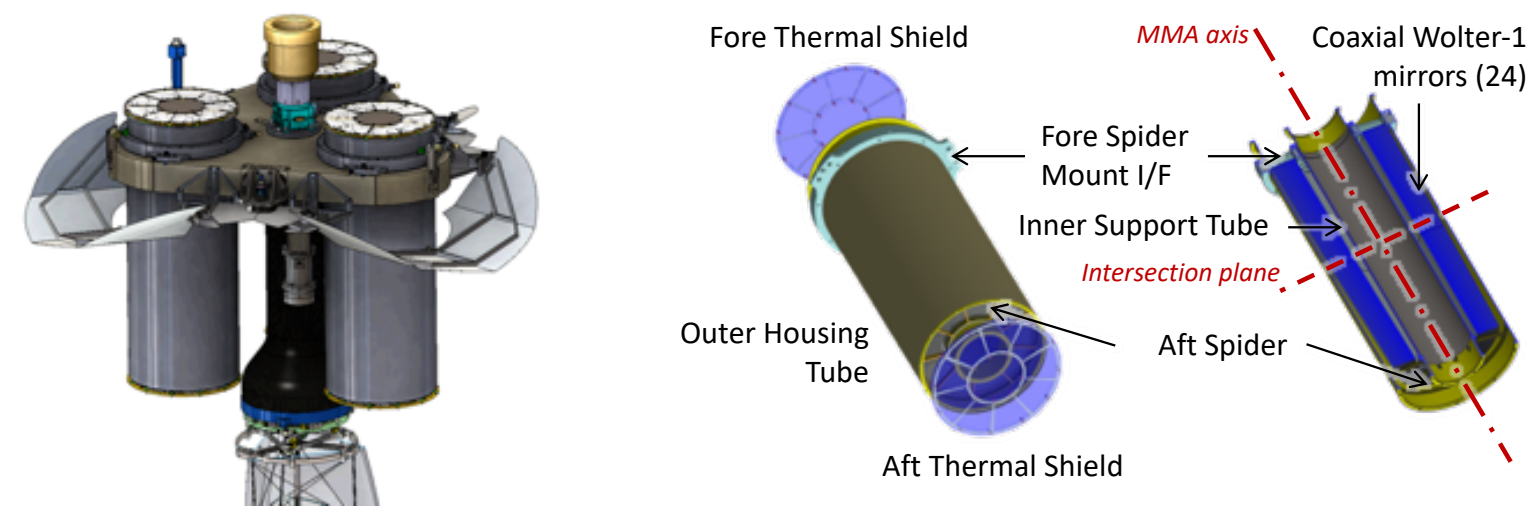

Figure 5. IXPE x-ray optics, comprising three (3) identical Mirror Module Assemblies (MMAs). Left panel shows the 3 MMAs mounted into support structure, along with other payload elements. Center panel displays an expanded view of an MMA - including fore and aft thermal shields, fore and aft spiders, and outer housing. Right panel exhibits a cut-away viewincluding the 24 (two-reflection) x-ray shells and inner support tube.

\subsubsection{Instrument}

Figure 6 displays the IXPE x-ray instrument—-three identical Detector Units (DUs) and a Detectors Service Unit (DSU)— contributed by INAF-IAPS and INFN and funded by ASI. The upper left panel depicts the 3 DUs mounted on the fore side of the top deck of the spacecraft, clocked at $120^{\circ}$ intervals and positioned to allow alignment with the 3 MMAs (§3.1.1). Each DU is mounted on and thermally connected to an individual interface plate, which is thermally isolated from the top deck and directly coupled to a shared radiator at the aft end of the Observatory ( $\$ 3.1 .3$ and Figure 4).
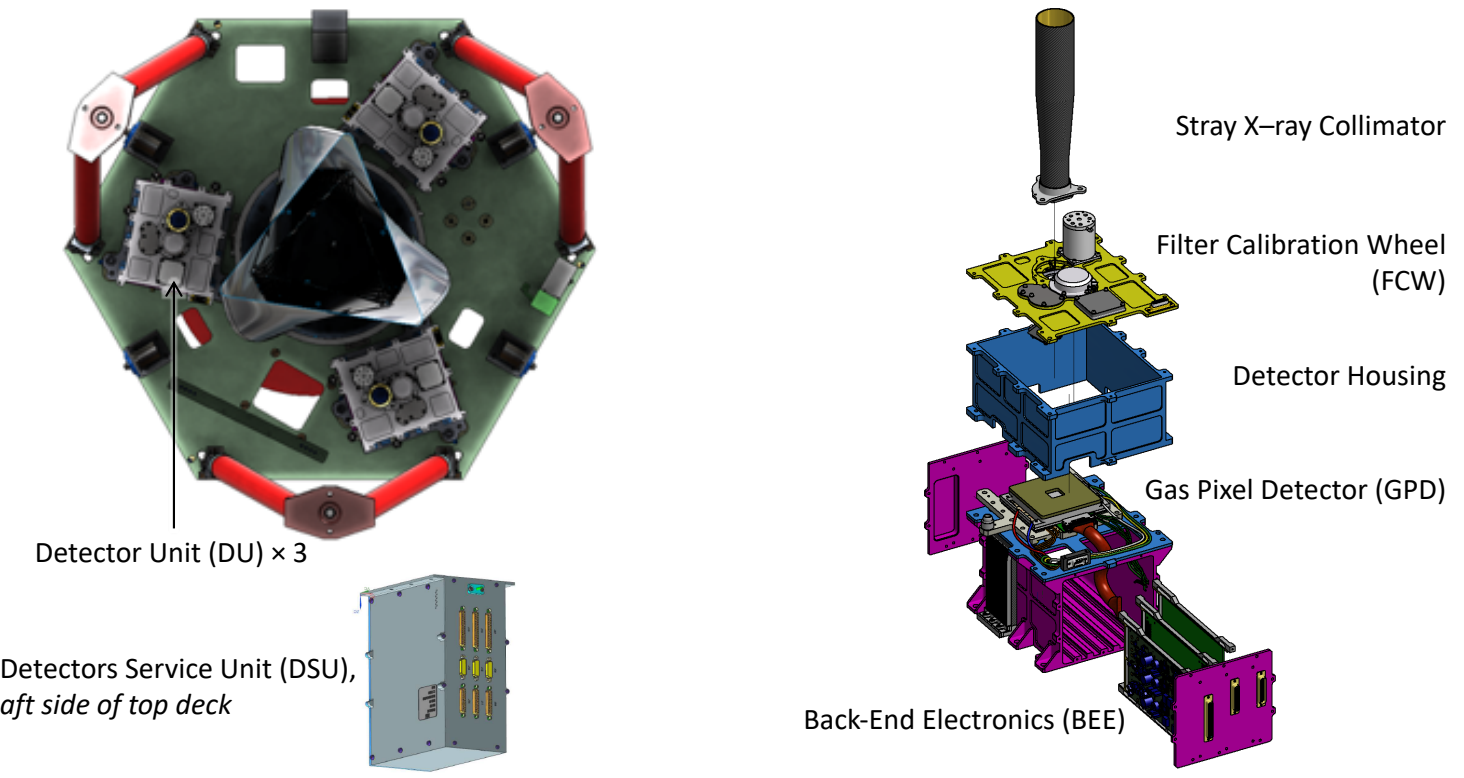

Figure 6. IXPE x-ray instrument, comprising three (3) identical polarization-sensitive Detector Units (DUs) and a Detectors Service Unit (DSU). Upper left panel shows the placement and clocking of the 3 DUs on the spacecraft's top deck; lower left panel displays the DSU, which is mounted on the aft side of the top deck. Right panel illustrates the DU componentsCollimator, Filter Calibration Wheel (FCW), Detector Housing, Gas Pixel Detector (GPD), and Back-End Electronics (BEE).

The DSU, mounted on the aft side of the top deck, controls all 3 DUs - including time management, power supply, and thermal control of the detectors. Further, the DSU acquires, consolidates, and transmits science and housekeeping telemetry from the DUs to the spacecraft's Integrated Avionics Unit (IAU, §3.2). Conversely, the DSU and receives ground commands through the IAU. 
The right panel of Figure 6 provides an expanded view of an IXPE DU, with key components labeled. Used in conjunction with the deployed x-ray shields ( $\$ 3.1 .3$ and Figure 4), the collimator prevents non-imaged $\mathrm{x}$ rays from reaching the Gas Pixel Detector (GPD). The Filter Calibration Wheel (FCW) provides seven positions ( $\$ 3.1 .4$ and Figure 8): an open position for standard science operations; an attenuating filter for viewing very bright $\mathrm{x}$-ray sources; a closed position; and four calibration sources containing iron-55 $\left({ }^{55} \mathrm{Fe}\right)$, one of which uses a crystal to produce polarized lines. The detector housing encloses the GPD, which detects the x-ray events and associated polarization information, as described below. The Back-End Electronics (BEE) distributes low- and high-voltage power to the GPD, receives and digitizes event data from the GPD, and pre-processes those data before transmitting to the DSU.
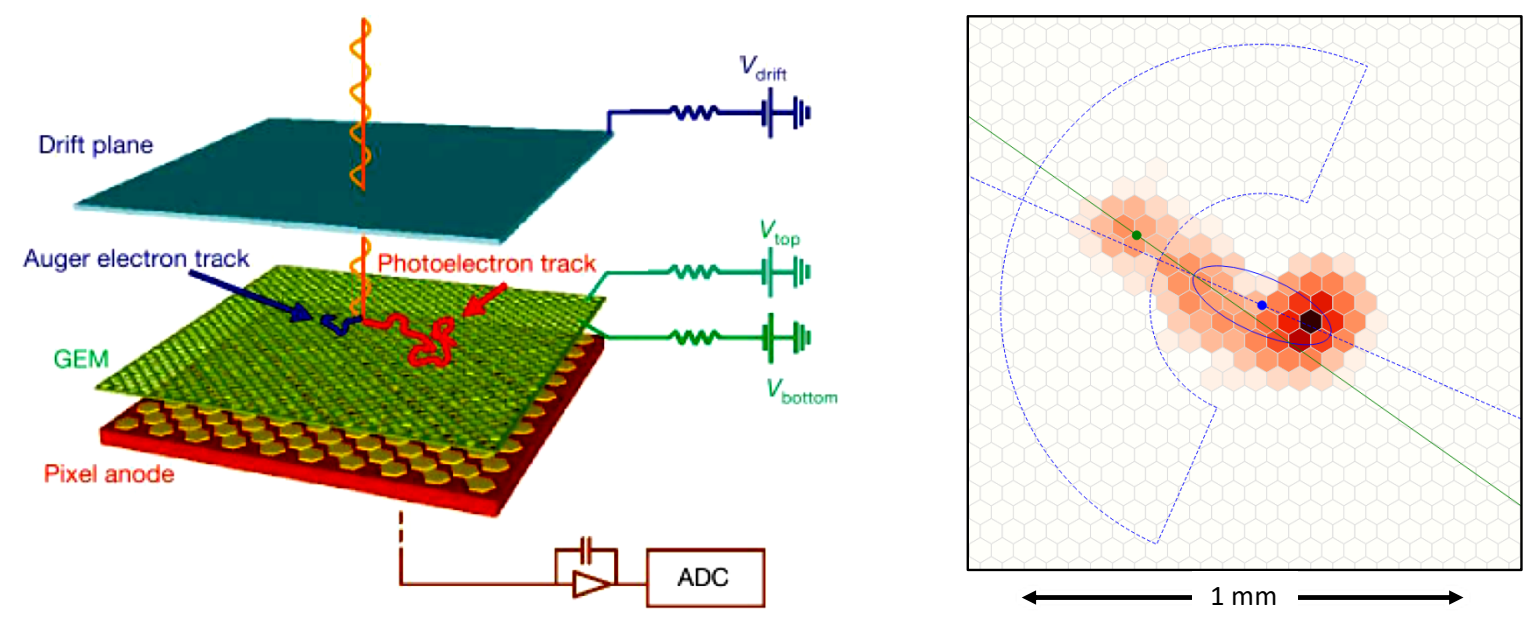

Figure 7. Operation of the Gas Pixel Detector (GPD) as a polarization-sensitive instrument. Left panel shows the essential components of the GPD and illustrates its response to a photo-absorbed x ray-namely, an ionization track of electrons. Right panel displays an actual ionization track resulting from photo-absorption of a 5.9-keV x ray, as imaged on the pixelated anode.

At the heart of the DU is the GPD, ${ }^{6}$ a small imaging gas proportional counter invented ${ }^{7}$ and developed ${ }^{8,9}$ by the Italian detector team. For IXPE, the fill gas is a mixture of dimethyl ether (DME, 80\%) and helium (He, 20\%) at 1 atmosphere, in a 10-mm-deep volume. The left panel of Figure 7 illustrates the principle of operation: An x ray absorbed in the drift region ejects a photoelectron, which loses energy as it collisionally ionizes the gas, producing an ionization track of electrons. This ionization track drifts to the GEM, where the strong electric field effects an avalanche that multiplies the number of electrons by a large factor (gas gain $\approx 500$ for the IXPE GEM). The amplified electron ionization track then transfers onto the pixelated anode, which is a $300 \times 352$ array of hexagonal pixels at $50-\mu \mathrm{m}$ pitch (same pitch and alignment as the holes through the GEM). This anode array is integral to an Application Specific Integrated Circuit (ASIC), which self-triggers on events, defines a region of interest (ROI), and transfers the ROI with the (analog) track image ${ }^{10}$ to the BEE. The BEE digitizes the track image, time tags the event, and transfers the data to the DSU for additional processing and consolidation before being telemetered through the IAU to the ground station.

The right panel of Figure 7 exhibits an actual GPD ionization track, similar to the data that IXPE will process on the ground. Processing algorithms applied to the ionization track extract the location on the detector where the photon was absorbed, its energy (proportional to the total charge in the track), and the initial direction of the photoelectron (correlated to the $\mathrm{x}$-ray electric-field direction). The modulation amplitude of a histogram of photo-ejection directions for a large number of $\mathrm{x}$-ray events is proportional to the polarization degree, through the energy-dependent modulation factor $\mu(E)$.

\subsubsection{Other payload elements}

Each MMA (\$3.1.1) and its corresponding DU (\$3.1.2) constitute an x-ray telescope. These 3 telescopes, the DSU, and the other payload elements (Figure 4) provided and integrated by Ball, comprise the full Telescope System. Beginning with structural payload elements, the Mirror Module Support Structure (MMSS) holds the 3 MMAs in their prescribed locations and aligned to the fore star tracker (a spacecraft component). In the stowed configuration (Figure 3 left panel), the 3 MMSS Bipods support the MMSS through launch, until the MMSS Launch Locks are released prior to deployment of the Boom. The Deployable Boom - from Northrop Grumman Innovation Systems (NGIS, formerly Orbital-ATK)connects the spacecraft's top deck to the MMSS through the MMSS Center Tube, serving as the shared optical bench. 
Alignment of each DUs with its respective MMA is critical as it determines the optical axis (or line of sight) of each x-ray telescope. Hence, the 3 DUs will be carefully aligned at Ball (\$3.1.5) to have the same relative positions as those of the 3 MMAs. However, upon deployment of the boom, the set of 3 DUs will likely be collectively displaced in translation and rotation (twist about the $Z$ axis) from the set of 3 MMAs. Accordingly, IXPE incorporates a Tip-Tilt-Rotate (TTR) Mechanism - from NGIS and essentially the same as that used on NuSTAR - between the Boom and the MMSS Center Tube. The TTR will be used during the commissioning phase (and occasionally during normal science operations, if needed) to perform this collective alignment.

Factors such as day-night thermal variations will slightly deform the Boom, collectively displacing the set of 3 DUs from the set of 3 MMAs. The Boom's Thermal Sock largely mitigates time-dependent distortion of the Boom-i.e., optical bench. However, to improve the knowledge of the Observatory's line of sight, ground processing will utilize time-tagged data from the IXPE Metrology System (Camera and LED-array Target) to correct the aspect solution ex post facto for each detected $\mathrm{x}$ ray.

The remaining payload components of the Telescope System support operation of the Instrument, as mentioned earlier (\$3.1.2). The deployed X-ray Shields combined with the DU Collimator prevent non-imaged $\mathrm{x}$ rays from reaching the Gas Pixel Detector (GPD). The shared Radiator at the aft end of the Observatory supports thermal control of each DU's GPD, being thermally coupled through an interface plate and conductive straps.

\subsubsection{X-ray calibration}

An extensive $\mathrm{x}$-ray calibration is planned ${ }^{11}$ for the IXPE MMAs and DUs ( 3 flight +1 spare, each), both separately and combined as telescopes, using polarized and unpolarized x-ray sources. Figure 8 depicts some of the ground support equipment (GSE), flight components, and facilities to be used for the x-ray testing and calibration.
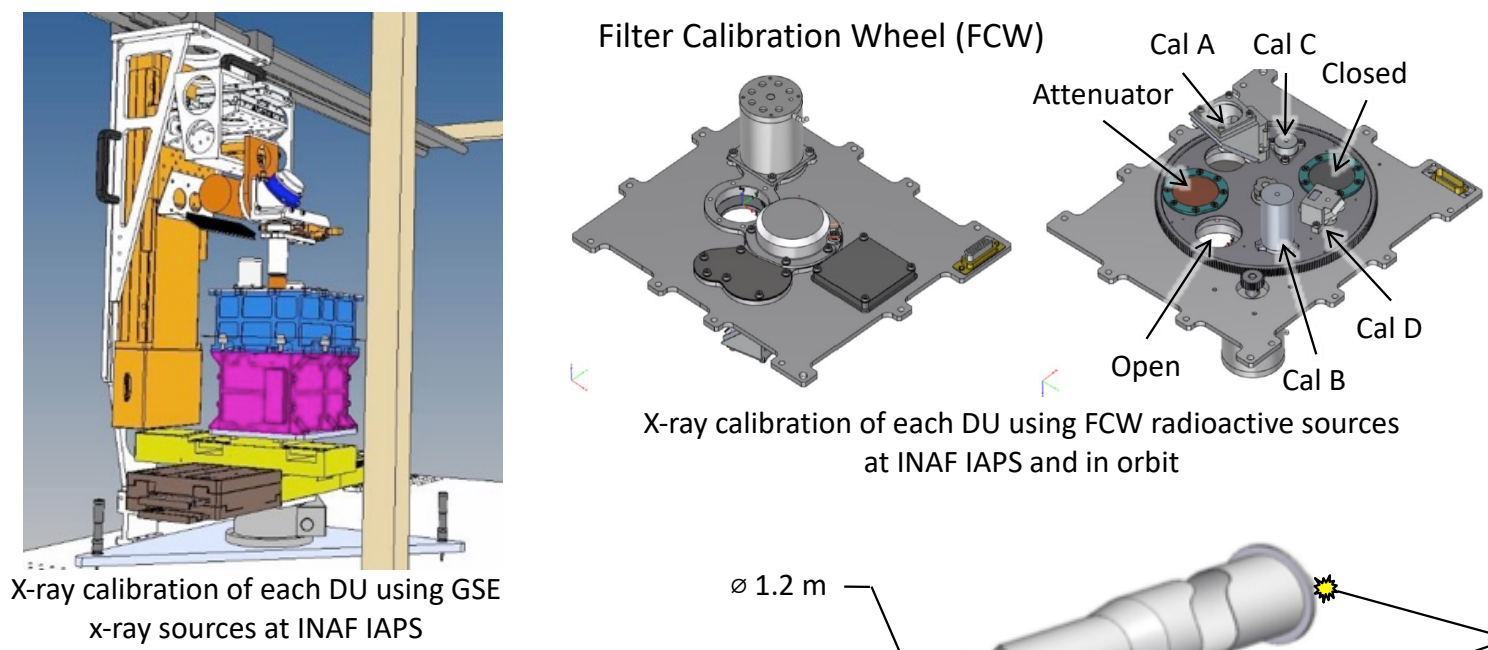

X-ray calibration of each DU using FCW radioactive sources at INAF IAPS and in orbit

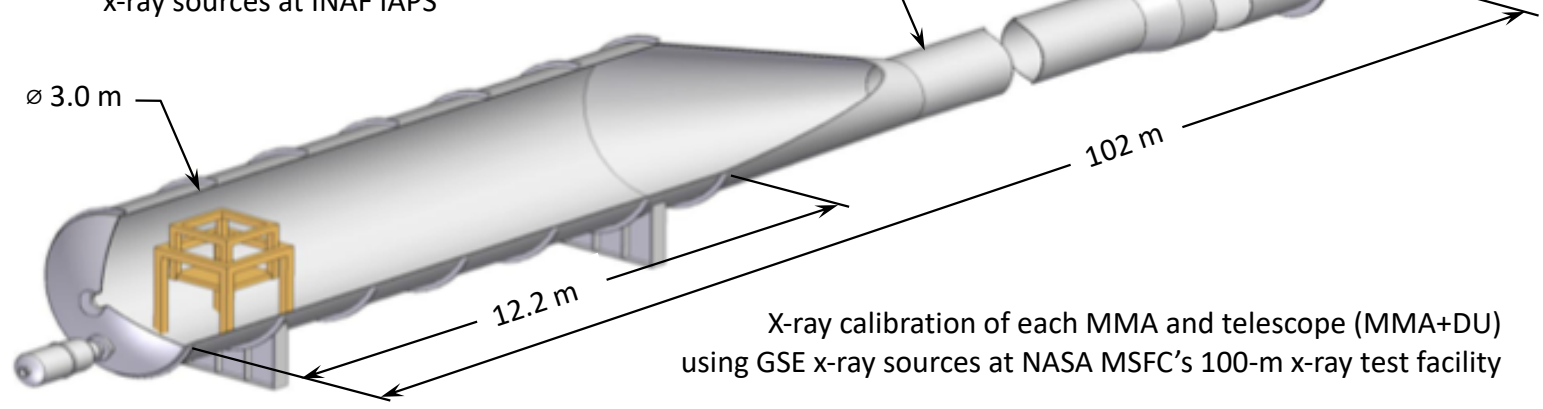

Figure 8. X-ray testing and calibration. Upper left panel portrays the Instrument Calibration Equipment (ICE) to be used for characterizing and calibrating the performance of the DUs at INAF IAPS. Upper right panel displays the top and bottom of the Filter Calibration Wheel (FCW) built by OHB-I and configured by INAF IAPS to include four radioactive $\left({ }^{55} \mathrm{Fe}\right)$ calibration sources. Lower panel shows a sketch of the 100-m x-ray test facility ("Stray Light Facility") at NASA MSFC. 
INAF-IAPS will calibrate ${ }^{12}$ each DU using its custom Instrument Calibration Equipment (ICE, Figure 8 upper left panel) in an IXPE-dedicated cleanroom. The ICE includes polarized and unpolarized x-ray sources, reference detectors, several stages that position and orient the detector and the source separately, and electrical and data interfaces to the DU. The DU calibration will measure, often for multiple x-ray energies or locations on the detector, the following:

1. Detection efficiency (probability of detecting an impinging $\mathrm{x}$ ray)

2. Spatial resolution

3. Modulation factor (ratio of modulation amplitude to polarization degree)

4. Limit on spurious polarization (false modulation of an unpolarized source)

5. Systematic accuracy of polarization-angle determination

6. Gain map

MSFC will calibrate each MMA using GSE x-ray sources and detectors at its 100-m x-ray test facility (Figure 8 lower panel). The MMA calibration will measure, typically for several x-ray energies, the following:

1. MMA effective area, on axis and several off-axis angles

2. MMA point spread function and its half-power diameter (HPD), on axis and at several off-axis angles

3. Ghost (not two-reflection) rays from a source outside the field of view

4. Focal length, derived using object distance from optical node to detector at best focus

With some minor analysis, the calibration of a telescope (MMA + DU) can be synthesized from the separate MMA and DU calibrations. To verify this synthesis, MSFC and the Italian Instrument Team will calibrate the 4 ( 3 flight +1 spare) telescopes at the 100-m facility. To accomplish this, MSFC will provide polarized x-ray sources, in addition to unpolarized sources used for the MMA calibration. The Telescope calibration will measure, typically for a subset of energies and positions used in the separate DU and MMA calibrations, the following:

1. Telescope effective area, on axis and a few off-axis angles

2. Telescope point spread function and its half-power diameter (HPD), on axis and at several off-axis angles

3. Ghost (not two-reflection) rays from a source outside the field of view

4. Focal length, derived using object distance from optical node to DU node at best focus

5. Modulation factor (ratio of modulation amplitude to polarization degree) for Telescope

6. Limit on spurious polarization (false modulation of an unpolarized source) for Telescope

NB: Polarization-related properties - modulation factor and spurious polarization (if any) - for the Telescope should be nearly identical to those for the $\mathrm{DU}$, as grazing-incidence reflectance is effectively independent of polarization.

In order to monitor the on-orbit performance of the IXPE Instrument, each DU has an FCW (Figure 8 upper right panel) that includes 4 radioactive calibration sources - along with open, attenuator, and closed positions. During earth occultation of a celestial x-ray target (about $40 \%$ of the orbit for most targets), one radioactive calibration source will be positioned to illuminate the GPD of one DUs. Over time, all 3 DUs will be exposed to all 4 calibration sources - one of which is polarized - and thus monitored throughout IXPE science operations. These FCW calibrations of each DU will measure the following:

1. Modulation factor (ratio of modulation amplitude to polarization degree)

2. Limit on spurious polarization (false modulation of an unpolarized source)

3. Gain map

The final category of calibration is parasitic to the IXPE observing program. Observations of celestial point sources monitor the point-spread function; comparisons of IXPE observations with those of other x-ray observatories-Chandra, XMM-Newton, etc.- provide cross-calibrations of non-polarimetric properties.

\subsubsection{Alignment}

After MSFC and the Italian Instrument Team complete calibration of the flight Telescopes at the 100-m x-ray facility, the MMAs and DUs are transported to Ball for alignment, integration, and testing. As alignment is crucial to optimal performance of the Observatory, Ball personnel will have rehearsed some alignment procedures using their alignment instrumentation during the $\mathrm{x}$-ray calibration campaign at MSFC. However, the permanent alignments occur during payload integration at Ball. 
The most demanding alignment requirement is to ensure that the triangle formed by the optical nodes of the 3 MMAs ("MMA nodes") $)^{\dagger}$ is congruent with that formed by the detection centers of the GPDs ("DU nodes"). If this is achieved, then the optical axes of all three Telescopes - the lines of sight determined by the MMA and DU nodes - can be simultaneously aligned to the optical axis of the Fore Star Tracker, through collective translations and rotation of the DU array to the MMA array. This, of course, is the function of the Tip-Tilt-Rotate (TTR) Mechanism (§3.1.3).

Note that it is co-alignment of the optical axes of the Telescopes — not of the MMAs (Figure 5 right panel) — that ensures maximum overlap of the celestial field-of view of the Telescopes. Nonetheless, alignment of the MMA axis to the Telescope optical axis is highly desirable, in that it improves image quality and throughput.

Ideally then, the 3 MMAs and the Fore Star Tracker are mounted on the MMSS, such that their (line-of-sight) axes are mutually parallel. If the 3 DU nodes are congruent to the 3 MMA nodes, the 3 Telescope optical axes can be co-aligned with the Star Tracker optical axis, through translations and rotation of the bus relative to the MMSS - e.g., using the TTR.

\subsection{Spacecraft}

Figure 9 shows the IXPE spacecraft bus and some of its components. The IXPE bus includes typical subsystems: Mechanical; Structural; Thermal; Power; Harness \& Electrical; Telecommunications; Command \& Data Handling; Attitude Determination \& Control; and Flight Software. Note that the IXPE Observatory has no Propulsion subsystem.

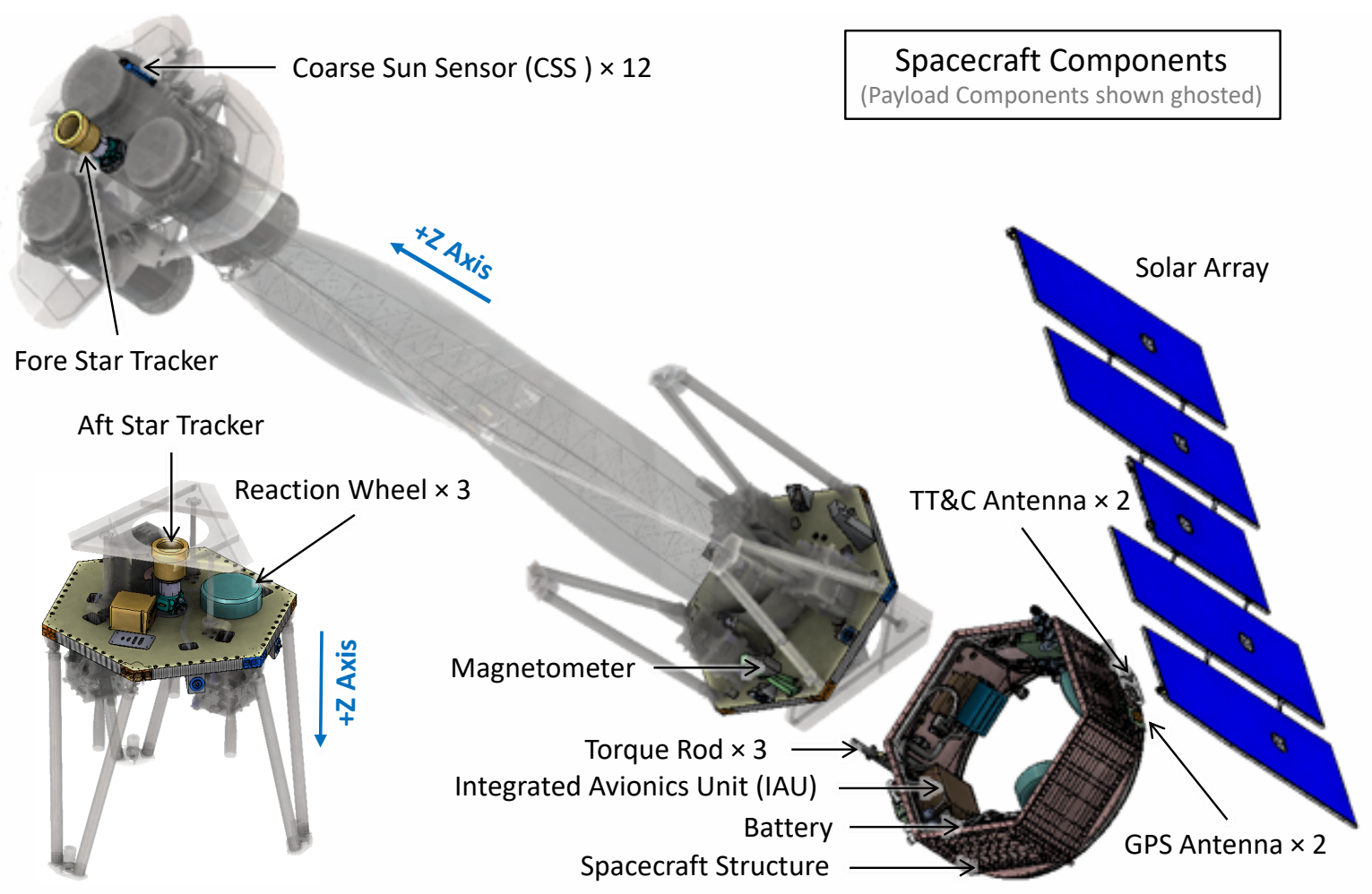

Figure 9. IXPE spacecraft bus. Spacecraft bus components are labeled; payload components are shown ghosted.

† The "MMA node" is the optical node of the Wolter-1 mirror system, which lies on the MMA optical axis (ideally the axis of cylindrical symmetry), at an axial position close to the intersection plane between the primary (parabolic) and secondary (hyperbolic) reflective surfaces (Figure 5 right panel). Recall that any rotation of an optic about its node leaves the position (but not necessarily the quality) of the image unchanged.

* The "DU node" is the point at mid depth in the GPD gas volume above the center of the pixelated anode (Figure 7). For best focus, the DU and MMA nodes should be separated by the MMA's focal length (Figure 3 right panel). 
The aforementioned subsystems provide the functionality to operate the bus as a spacecraft and to support operation of the payload as a Telescope System. This section focuses on those subsystems that are especially tailored to the specific needs of the IXPE Observatory_-Attitude Determination \& Control (§3.2.1) and Command \& Data Handling (§3.2.2).

\subsubsection{Attitude Determination \& Control Subsystem (ADCS)}

The ADCS provides 3-axis stabilized pointing - enabling slewing, acquiring, and staring at selected celestial targets for extended periods - to an expected (a priori) accuracy of about 25 arcseconds (99\% circular error probability, CEP 99$)$. In addition, it acquires precision time and position from the Global Positioning System (GPS), for accurate time-tagging of science and housekeeping data throughout the Observatory. The ACDS performs momentum management using magnetic torquers to unload the reaction wheels against the earth's magnetic field, obviating the need for a propulsion subsystem.

Figure 9 identifies Spacecraft components supporting the ADCS functions:

1. Fore $(+Z)$ and Aft $(-Z)$ Star Trackers -2 optical heads with a shared electronics unit

2. Coarse Sun Sensors-12 sensors, distributed over the Observatory for omnidirectional coverage

3. Magnetometer-1 3-axis unit

4. Torque Rods-3 magnetic torquers, orthogonally oriented

5. Reaction Wheels - 3 wheel assemblies, orthogonally oriented

6. GPS components - 1 receiver with 2 antennas, for nearly continuous GPS coverage

As with most IXPE Spacecraft subsystems, ADCS functions are administered by the Integrated Avionics Unit (IAU), which provides all the functionality for the Command \& Data Handling Subsystem.

\subsubsection{Command \& Data Handling Subsystem (CDHS)}

Through its IAU, the CDHS supplies computational services, memory management, data handling, telemetry management, and time messaging both for the bus and for the payload. Driven by flight software hosted on its computer, the CDHS monitors and controls the ADC, Telecommunications, Electrical Power, and Thermal subsystems. In addition, the CDHS communicates directly with the Instrument’s (§3.1.2) Detectors Service Unit (DSU), forwarding commands and receiving and packaging science and housekeeping data from the DSU for transmission to the ground station (§4.3) with the rest of the telemetered data.

\section{GROUND SYSTEM}

Figure 10 indicates the structure of the IXPE ground system. At the top level, the ground system has three branchesscience operations ( $\$ 4.1)$, mission operations ( $\$ 4.2)$, and telecommunications ( 44.3$)$.

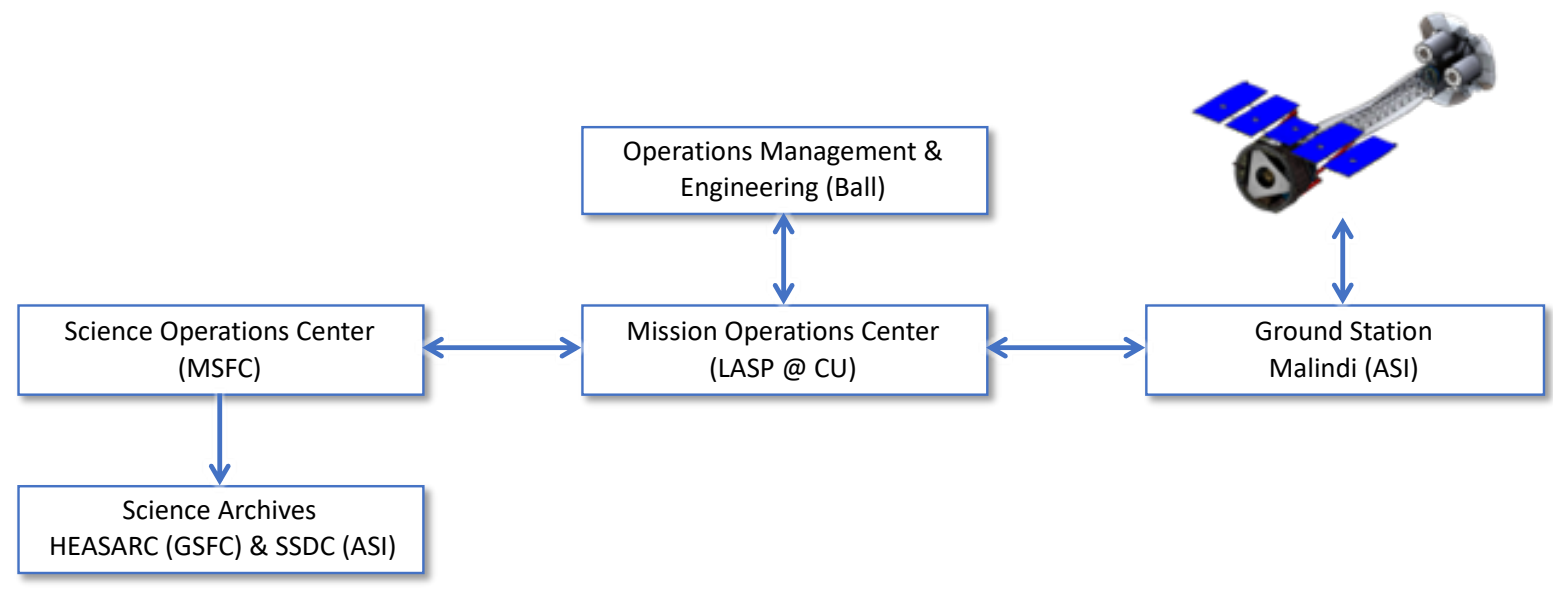

Figure 10. IXPE ground system. This simplified schematic diagram of the Ground System highlights science operations (left), mission operations (center), and telecommunications (right). 


\subsection{Science Operations}

IXPE science operations are the responsibility of the Science Operations Center (SOC) at MSFC, with support from the ASI Space Science Data Center (SSDC). The SOC is charged with design, implementation, and execution of the software tools for science data processing and basic analysis of IXPE observations. This includes development and maintenance of the calibration database (CALDB).

During phase E (operations), the SOC interfaces with the Mission Operations Center (§4.2) in two roles. First, based upon input from the IXPE Science Community, the SOC develops a science observing program that it transmits to the MOC for detailed scheduling of mission operations. Second, the SOC receives from the MOC all Observatory data (science, housekeeping, engineering, and ancillary) data needed to produce the science data products for each observation.

For each science observation, the fundamental data product produced by the SOC is a time-tagged event list. Based upon the time-tagged ionization tracks from the Instrument, SOC processing first determines the time, detector coordinates, (pulse-invariant) energy, and photo-ejection direction (on the detector) for each detected $\mathrm{x}$ ray within an observation. It then employs time-tagged ADCS and Metrology-System data to map detector coordinates into celestial coordinates.

Within a week of an IXPE observation, the SOC transmits all data products for that observation to NASA's High-Energy Astrophysics Science Archive Research Center (HEASARC) at GSFC, for public release. ASI's Space Science Data Center will mirror the IXPE data archived at the HEASARC.

\subsection{Mission Operations}

IXPE mission operations are the responsibility of Ball Aerospace, which oversees IXPE activities at the Mission Operations Center, run by the Laboratory for Atmospheric and Space Physics (LASP) at the University of Colorado (CU). Ball engineers support mission operations in monitoring and assessing technical performance of the Observatory. Besides interacting closely with Ball, the MOC interfaces with the SOC (\$4.1) and telecommunicates with the Observatory through ASINet Fucino Gateway connected to the Malindi ground station (§4.3).

The MOC develops a detailed timeline of science observations (including calibrations) and spacecraft events (slews, telecommunications, etc.) into a command load, considering various operational constraints. Command loads are transmitted to the Observatory through the ground station. Upon receiving downlinked telemetry from the Observatory through the ground station, the MOC processes and distributes those data to the SOC and the housekeeping and engineering data to the Ball technical team.

\subsection{Telecommunications}

ASI contributes use of its Malindi (Kenya) ground station for S-band communication with the IXPE Observatory. The ASINet Fucino Gateway connects the Malindi ground station to the IXPE MOC. The MOC and ASI coordinate scheduling the ground contacts with the IXPE Observatory. For an equatorial orbit, the Malindi location $\left(3^{\circ} \mathrm{S}\right.$ latitude) provides excellent visibility - up to 14 contacts a day. Nominally, commanding passes will occur one day a week; telemetry downlinking passes are scheduled based upon anticipated data volume.

A commercial ground station in Singapore ( $1^{\circ} \mathrm{N}$ latitude), part of NASA's Near-Earth Network (NEN), serves as a backup station to Malindi. For some operations early in the mission or during anomalies, the IXPE Observatory will communicate with the MOC through NASA's Tracking and Data Relay Satellite System (TDRSS), which can provide near-continuous communication.

\section{ACKNOWLEDGEMENTS}

IXPE is a NASA Astrophysics Small Explorers (SMEX) mission, managed by MSFC and overseen by the Explorers Program Office at GSFC. The Italian contribution to the IXPE mission is supported by the Italian Space Agency through agreement ASI-INAF n.2017-12-H.0 and ASI-INFN agreement n.2017-13-H.0. 


\section{REFERENCES}

[1] Weisskopf, M. C., Ramsey, B., O'Dell, S., Tennant, A., Elsner, R., Soffitta, P., Bellazzini, R., Costa, E., Kolodziejczak, J., Kaspi, V., Muleri, F., Marshall, H., Matt, G., \& Romani, R., "The Imaging X-ray Polarimetry Explorer (IXPE)," SPIE 9905, 17 10pp (2016).

[2] Soffitta, P., "IXPE the Imaging X-ray Polarimetry Explorer," SPIE 10397, 0I 9pp (2017).

[3] Weisskopf, M. C., Cohen, G. G., Kestenbaum, H. L., Long, K. S., Novick, R., \& Wolff, R. S., "Measurement of the X-ray polarization of the Crab Nebula," ApJ 208, L125-L128 (1976).

[4] Weisskopf, M. C., Silver, E. H., Kestenbaum, H. L., Long, K. S., \& Novick, R., "A precision measurement of the Xray polarization of the Crab Nebula without pulsar contamination," ApJ 220, L117-L121 (1978).

[5] Ramsey, B. D., "Optics for the Imaging X-ray Polarimetry Explorer," SPIE 10399, 07 10pp (2017).

[6] Sgrò, C., "The gas pixel detector on board the IXPE mission," SPIE 10397, 0F 7pp (2017).

[7] Costa, E., Soffitta, P., Bellazzini, R., Brez, A., Lumb, N., \& Spandre, G., "An efficient photoelectric X-ray polarimeter for the study of black holes and neutron stars," Nature 411, 662-665 (2001).

[8] Bellazzini, R., Spandre, G., Minuti, M., Baldini, L., Brez, A., Cavalca, F., Latronico, L., Omodei, N., Massai, M. M., Sgro', C., Costa, E., Soffitta, P., Krummenacher, F., \& de Oliveira, R., "Direct reading of charge multipliers with a self-triggering CMOS analog chip with $105 \mathrm{k}$ pixels at $50 \mu \mathrm{m}$ pitch," NIMPA 566, 552-562 (2006).

[9] Bellazzini, R., Spandre, G., Minuti, M., Baldini, L., Brez, A., Latronico, L., Omodei, N., Razzano, M., Massai, M. M., Pesce-Rollins, M., Sgr\&oacute;, C., Costa, E., Soffitta, P., Sipila, H., \& Lempinen, E., "A sealed Gas Pixel Detector for X-ray astronomy," NIMPA 579, 853-858 (2007).

[10] Nasimi, H., "Overview of the detector and its readout on board the Imaging X-ray Polarimetry Explorer," SPIE 10699, 5D 8 pp (2018).

[11] Muleri, F., Baldini, L., Baumgartner, W., Evangelista, Y., Fabiani, S., Kolodziejczak, J., Latronico, L., Lefevre, C., O'Dell, S. L., Ramsey, B., Sgrò, C., Soffitta, P., Tennant, A., \& Weisskopf, M. C., "Calibrating the IXPE observatory from ground to space," SPIE 10397, 0G 8pp (2017).

[12] Muleri, F., Lefevre, C., Piazzolla, R., Morbidini, A., Amici, F., Attina, P., Centrone, M., Del Monte, E., Di Cosimo, S., Di Persio, G., Evangelista, Y., Fabiani, S., Ferrazzoli, R., Loffredo, P., Maiolo, L., Maita, F., Primicino, L., Rankin, J., Rubini, A., Santoli, F., Soffitta, P., Tobia, A., Tortosa, A., \& Trois, A., "Calibration of the IXPE instrument," SPIE 10699, 5C 11pp (2018). 